
Methods and Applications

Research Article

\title{
Novel Adaptive Decision Threshold Modulation Technique for UWB Direct Chaotic Communications
}

\author{
Said Sadoudi ${ }^{1}$, Caml Tanougast ${ }^{* 2}$ and Mohamad Salah Azzaz ${ }^{1}$ \\ ${ }^{1}$ Laboratoire Systèmes de Communications (LSC), Ecole Militaire Polytechnique, Algiers, Algeria. \\ ${ }^{2}$ Laboratoire de Conception, Optimisation et Modélisation des Systèmes (LCOMS), Lorraine University, Metz, France.
}

Received 19 September 2014; Revised 14 October 2014; Accepted 25 November 2014

\begin{abstract}
A new non-coherent chaotic modulation technique based on adaptive decision threshold is proposed for the UltraWideBand (UWB) Direct Chaotic Communication (DCC) technology. The principal advantages of the proposed technique are: (1) Removing the threshold problem of the classical Chaotic On-Off Keying modulation technique which uses a nonzero decision threshold; (2) Providing a high throughput comparing to the others techniques since it do not uses any delay at the modulation; (3) Reducing the transmitted power, thanks to a transmitted bit energy devised by two. The obtained simulation results show high Bit Error Rate performances of the proposed technique applied in an UWB DCC system. In addition, the new chaotic modulation is more suitable in all DCC-based communications schemes.
\end{abstract}

Keywords: Chaotic modulation, Colpitts oscillator, UWB, DCC, COOK.

\section{Introduction}

In the early 2000s, researchers developed and experimentally demonstrated the feasibility to generate directly chaos in the microwave band [1-5]. The first Microwave Chaotic Oscillator (MCO), based on the Colpitts oscillator [6], was proposed and realized by Panas et al [1]; which generates chaotic signals in the frequency band 900 $1000 \mathrm{MHz}$. Later, several approaches have been proposed in order to broaden the bandwidth of the microwave chaotic signals [2-5].

This discovery was the starting point for the use of a chaotic signal directly as a carrier of information, and thus gave birth to the new chaos-based communication technologies called Direct Chaotic Communication (DCC) [7] and Direct Chaotic Radar (DCR) [8,9].

In the year 2002, the authors in [10] have presented for the first time the theory basics of the DCC technology. The motivation of this work is focalized in earlier works which have verified the practical applicability of this new chaotic communication technology. In this context, we cite the work presented in [11] and [12], where a wideband communication system operating in 900-1000 $\mathrm{MHz}$ band and providing transmission rates of 10 to $100 \mathrm{Mbps}$ was presented, and in [13], a results of experiments with UltraWideband Direct Chaotic Circuit (UWB DCC) operating in 500-3500 MHz band with up to $200 \mathrm{Mbps}$ rate are given.

* E-mail address: camel.tanougast@univ-lorraine.fr ISSN: 1791-2377 (c) 2015 Kavala Institute of Technology. All rights reserved.
Several chaotic modulation schemes can be applied in the DCC technology such as Chaotic On-Off Keying (COOK), Differential-Chaos Shift-Keying (DCSK), and Pulse-Position Modulation (PPM) [14]. However, in [14], the authors affirmed that the COOK modulation scheme offers several advantages for the UWB DCC technology. Firstly, it is one of the simplest modulation schemes. Indeed, its transceiver architecture has the less complexity, i.e., just a chaotic generator and a modulator at the $T_{X}$, and an envelope detector and a constant threshold estimator at the RX. Secondly, it has $3 \mathrm{~dB}$ more energy efficiency than DCSK and PPM, which can then provides additional power saving. In addition, they conclude that the UWB DCC-COOK system is a possible solution for the new IEEE 802.15.4a alternative PHY layer stand and then a good candidate for the low-cost, low-power and low-complexity LR-WPAN applications. However, the main disadvantage of this technique is that it requires nonzero threshold estimation, which degrades its performances [14].

In this paper, we propose a solution to overcome the problem of nonzero threshold of UWB DCC-COOK schemes and which enjoys the benefits of the classical COOK modulation. For this, we propose to use a new noncoherent receiver structure for the COOK modulation technique, where the decision threshold is estimated and chosen directly at the reception of each Chaotic Radio Pulse (CRP), contrary to the structure used in [14] where it is chosen previously. This means that, in the proposed original solution and for the general case, the decision threshold is chosen adaptively according to the noise energy 
contaminating the received CRP. Consequently, in the case of the UWB technology applications, where the CRP duration is very small (in the order of ns), the decision threshold of the proposed COOK receiver structure can be chosen zero. For validating our approach, applied in an UWB DCC system, we use a professional RF and microwave circuit simulator, the Agilents Advanced Design System (ADS) [15]. We have shown the Bit Error Rate (BER) performance improvements of our approach compared to those of well-known non-coherent modulation techniques such as classical COOK, DCSK [16], CDSK [17], and RD-DCSK [18].

The rest of this paper is organized as follows: Section 2 presents the principle of the DCC technology and its combination with the UWB technology. In Section 3 the details of the basic theory of the proposed chaotic modulation technique are given. Simulations and BER performance analysis, using the ADS simulator, of UWB DCC system based on the proposed approach are presented in Section 4. Finally, in Section 5 the appropriate conclusions are presented.

\section{DCC Principle}

In 2002, Dmitriev et al. presented and detailed the basis of the DCC theory [10]. This new technique of chaotic communication is based on three main ideas:

1. Chaotic source generates oscillations directly in the prescribed microwave band without the need of local oscillator and mixer;

2. Information is put into the chaotic signal by means of forming the corresponding sequence of chaotic radio pulses;

3. Information is retrieved from the microwave signal without intermediate heterodyning.

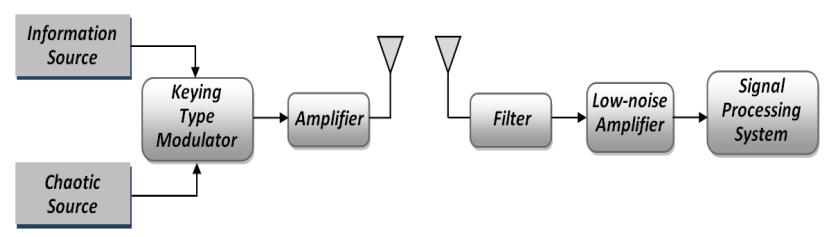

Fig. 1. Block diagram of the DCC system.

In Fig. 1, we present the block diagram of the DCC system using external modulation given by Dmitriev et al. [10]. The transmitter is composed of a chaotic source (MCO) that generates the chaotic carrier signal directly in the microwave band, an information source, a keying-type modulator, an amplifier and an antenna. The receiver is composed of a broadband antenna, a filter which permits the signal to pass the signal within the frequency band of the transmitter, a low-noise amplifier, and a signal processing system. This last can recover the information from the received signal by using non-coherent receiver by integrating the pulse power over the pulse interval, or by using coherent receiver by convolving the chaotic radio pulses with corresponding reference pulses generated in the receiver [10]. In the general case, the chaotic source generates a chaotic carrier in the microwave band with the following frequency bandwidth:
$\Delta F=F_{u}-F_{l}$

where $F_{u}$ and $F_{l}$ are the lower and upper boundaries of the chaotic oscillation band respectively, and while the central frequency is given by the following expression:

$F_{0}=\left(F_{u}+F_{l}\right) / 2$

\subsection{UWB DCC Concept}

The first application of the DCC technology is in the UWB technology [10]. The combination of the two technologies is feasible since it is possible experimentally to generate directly RF chaotic carrier from MCO [1], [2] and [3]. Therefore, the principle idea of the UWB DCC technology is resumed to replace the chaotic source of the transmitter presented in Fig. 1 by an UWB chaotic generator. This generator generates an UWB chaotic signal (carrier) satisfying the U.S. Federal Communications Commission (FCC) UWB regulations [14], i.e., a bandwidth of more than $7.5 \mathrm{GHz}$, particularly the bandwidth [3.1-10.6] GHz, can be exploited for unlicensed UWB applications. A principle scheme of the UWB DCC system with $\Delta F=7.5 \mathrm{GHz}, F_{u}=3.1$ $\mathrm{GHz}$ and $F_{l}=10.6 \mathrm{GHz}$ is presented and detailed in [14], where the authors have given the advantages of the UWB DCC in comparison with the conventional UWB technology. In fact, in an UWB DCC system, using for example the COOK modulation technique, the CRP is a fragment of the continuous chaotic carrier with duration $\tau_{c}$ and frequency bandwidth $\Delta F_{c}$, and has the following characteristics:

1. $\tau_{c}$ is longer than the quasi-period of the chaotic oscillations $T_{c}$.

2. $\Delta F_{c}$ is independent of the pulse duration $\tau_{c}$, but is determined by $\Delta F$ (Eq.1).

Thereby, from the above characteristics, the bandwidth of the CRP is defined as follows:

$\Delta F_{c}=\Delta F \neq \frac{1}{\tau_{c}}$

This means that the power spectrum of a stream of chaotic pulses will be equal to that of the UWB chaotic carrier (for more details see [10] and [14]). Consequently, the bandwidth is independent of $\tau_{c}$ contrary to the conventional original UWB systems where the relation between the pulse frequency bandwidth $\Delta F_{p}$ and the pulse duration $\tau_{p}$ is inversely proportional and defined by the following equation [14]:

$\Delta F_{p}=\frac{1}{\tau_{p}}$

\subsection{UWB Chaotic Generator}

The basic element of an UWB DCC system is the MCO which generates the chaotic signals directly in the desired microwave band. In this work, we use the well-known Colpitts oscillator which is widely investigated in literature [1-6]. Just for validation reasons, we simulate with ADS the basic configuration of this oscillator presented in [8], as it is shown in Fig. 2. From this figure, we note that the Colpitts oscillator contains a Bipolar Junction Transistor (BJT) as the 
gain element, which is the BFG505 whose threshold frequency is $9 \mathrm{GHz}$, and a resonant network consisting of an inductor and a pair of capacitors [8,9]. In our simulations, the circuit parameters values are listed as follows: $V_{1}=-V_{2}=$ $10 \mathrm{~V}, R_{1}=25 \mathrm{Ohm}, R_{2}=1.6 \mathrm{kOhm}, L=4.5 \mathrm{nH}, C_{1}=C_{2}=4$ $\mathrm{pF}$. With these values, we obtain the ADS simulation results of the MCO signals presented in Fig. 3.

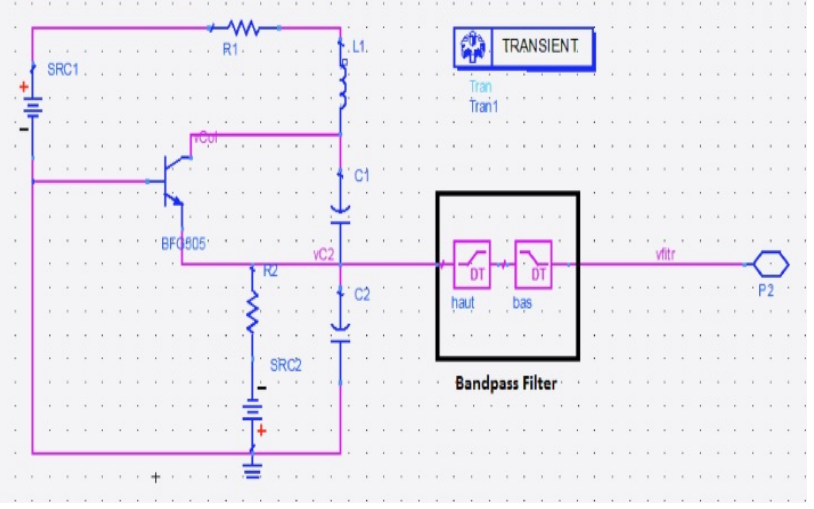

Fig. 2. ADS description of the MCO architecture.

In Fig. 3(a), the time-domain waveform of the voltage node $V_{C 2}$ (voltage across the capacitor $C_{2}$ ) is shown. The attractor of the circuit in the $V_{C 2}-V_{C o l}$ plane, where $V_{C o l}$ is the voltage at the collector of the transistor (voltage across the capacitor $C_{1}$ ) is shown in Fig. 3(b). In Fig. 3(c), the broadband continuous spectrum (about $15 \mathrm{GHz}$ ) of the signal $V_{C 2}$ is shown.
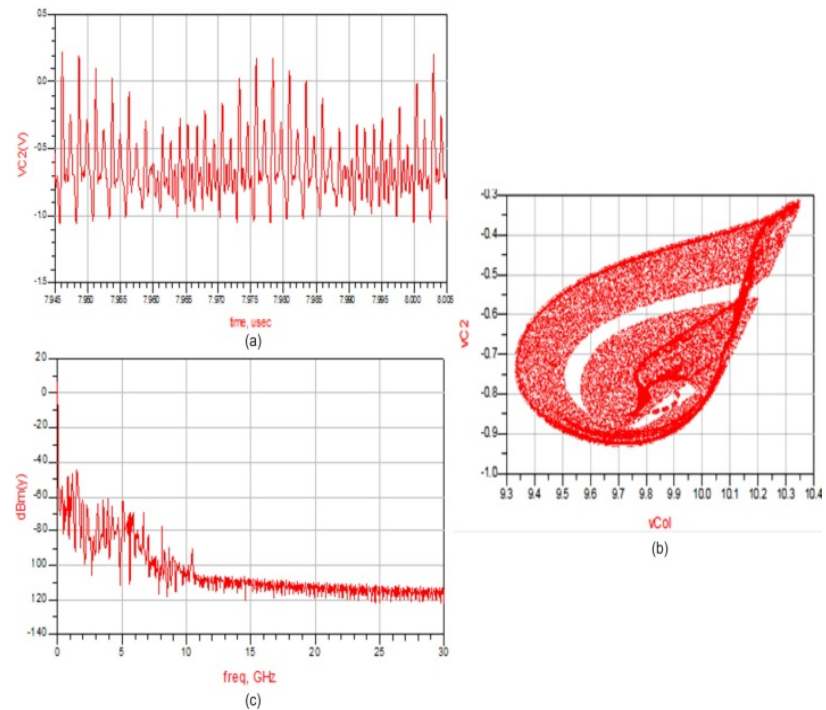

col

Fig. 3. ADS simulation results of the MCO signals before bandpass filtering: (a) Time-domain waveform of the voltage node $V_{C 2}$, (b) The chaotic attractor in the $V_{C 2}-V_{C o l}$ plane and (c) The spectrum of the signal $V_{C 2}$.

From the results of Fig. 3, we note that the bandwidth spectrum of the microwave chaotic signal generated by the simulated circuit is over $15 \mathrm{GHz}$ a limited decrease of power lower of $-41.3 \mathrm{dBm}$ from the maximal power signal. However, in this work, we need a chaotic signal (carrier) which satisfies the strict FCC UWB regulations resumed in a signal power lower than $-41.3 \mathrm{dBm}$ at the UWB frequency band [3.1-5] GHz. Knowing that for validation reason, we are interested only by the lower band $([3.1-5] \mathrm{GHz})$ of the FCC UWB band. For this, we have used a bandpass filter formed by two filters, a low pass filter having a cutoff frequency of $3.1 \mathrm{GHz}$ and a high pass filter having a cutoff frequency of $5 \mathrm{GHz}$ (see Fig. 3). The simulation results after the bandpass filtering of the microwave chaotic signal $V_{C 2}$ is given by Fig. 4 . The Fig. 4(a) shows the filtered signal $V_{C 2}$, i.e., UWB chaotic carrier while its spectrum is shown in Fig. 4(b) confirming that the obtained UWB chaotic carrier satisfies the FCC UWB regulations. In fact, the obtained bandwidth spectrum is from 3.1 to $5 \mathrm{GHz}$ with the power of $-47.306 \mathrm{dBm}$, as it is indicated by the pointer $m_{1}$ in Fig. 4 , which is lower than $-41.3 \mathrm{dBm}$. In summary, this UWB chaotic generator can be used as an UWB chaotic signal source in an UWB DCC system.
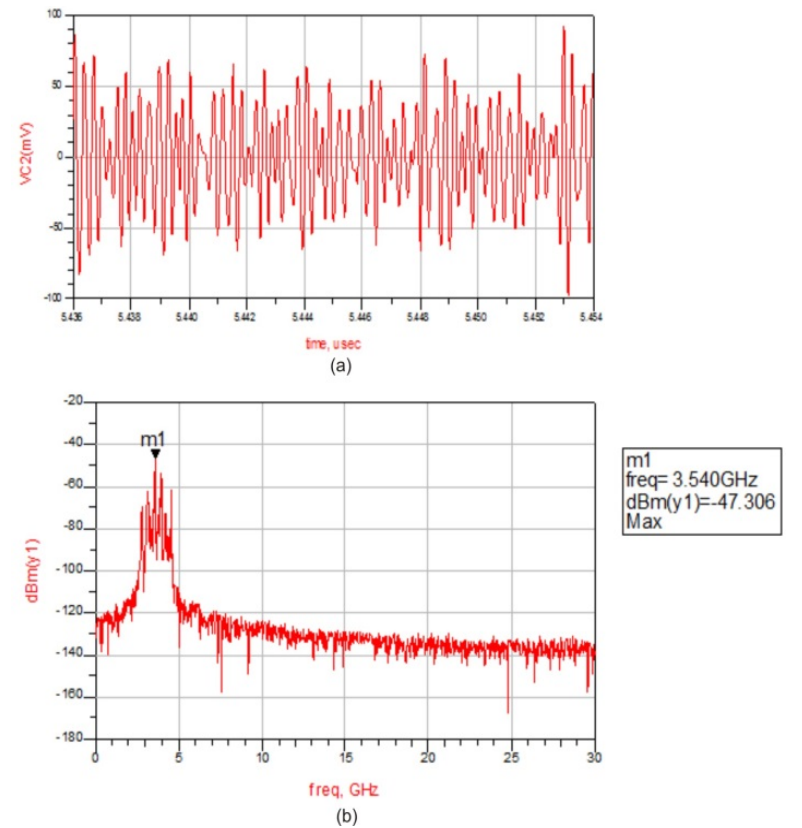

Fig. 4. ADS simulation results of the MCO signals after bandpass filtering: (a) The filtered signal $V_{C 2}$ and (b) The spectrum of the filtered signal $V_{C 2}$.

\section{The Proposed AT-COOK Modulation for UWB DCC Technology}

\subsection{The Idea}

In [10], Dmitriev et al. has affirmed that for the DCC technique, there are two possible manners to recover the information at the receiver, noncoherently (by using noncoherent receiver) or coherently (by using coherent receiver). In the presented work, we are interested by the non-coherent receiver. For this case, the authors in [10] have proposed two non-coherent receiver structures. The block diagrams of these structures are depicted in Fig.5. The difference between the two structures is in the location of the Threshold Unit versus the Processing Unit (see Fig.1) [10]. More precisely, suppose that the COOK modulation technique is applied in an UWB DCC system. The transmitted signal through a noisy channel is expressed as follows:

$y(t)=\left\{\begin{array}{l}y_{0}(t)+\eta(t), " 0 " \text { is transmitted } \\ y_{1}(t)+\eta(t), " 1 " \text { is transmitted }\end{array}\right.$

where $\eta(t)$ represents the additive Gaussian noise with spectral density $N_{0}$. At the receiver and after the envelope detection (demodulation block), the signal at the Threshold Unit is expressed by: 
$\left\{\begin{array}{l}d_{0}=\int_{0}^{T} \eta^{2}(t) d t, " 0 " \text { is transmitted } \\ d_{1}=\int_{0}^{T}\left(y_{1}(t)+\eta^{2}(t)\right)^{2} d t, " 1 " \text { is transmitted }\end{array}\right.$

Then, the decision of receiving " 0 " or " 1 " is taken by comparing the obtained value $d_{i}(i=0,1)$ with a predefined threshold value $d$. Therefore, for the two receiver structures presented in Fig. 5, Dmitriev et al. shows that in the first structure (Fig. 5(a)) corresponding to the receiver structure of the classical COOK technique, the threshold value $d$ is a predefined constant taken outside the Processing Unit. However, in the second structure (Fig. 5(b)), the decision threshold value $d$ is chosen according to estimated value of noise energy. Consequently, the decision threshold depends on the noise energy affecting the received signal and must be taken automatically inside the Processing Unit.

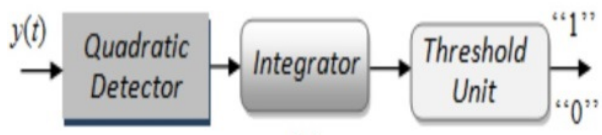

(a)

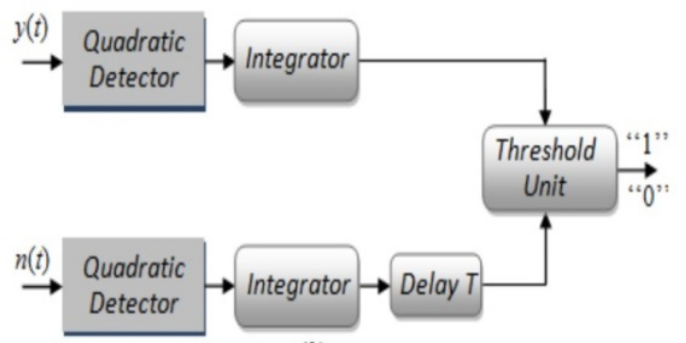

(b)

Fig. 5. Non-coherent receiver structures for DCC system.

In this work, we exploit and explore this last proposition of Dmitriev et al., the main originality of our proposed approach, which consists of using the second non-coherent receiver structure for the COOK-based UWB DCC system. Knowing that in their paper [10], they have proposed this idea only by giving a principle scheme without any development and performances analysis, and until now, this idea is not treated and applied for the UWB DCC technology in the literature, for the best of our knowledge. Indeed, from the affirmation in [10], that in the second structure (Fig. 5(b)) the decision threshold value must be taken automatically inside the processing unit, we can say, in other words, that the decision threshold value $d$ must be adapted to the estimated noise energy value. Thus, by considering his non-coherent receiver structure, we propose a new COOKbased modulation technique for the UWB DCC technology, called Adaptive Threshold COOK (AT-COOK). The principle of this new approach is presented in the next section.

\subsection{AT-COOK Principle}

The basic principle of the proposed AT-COOK is shown by the block diagram of Fig. 6. It enjoys the benefits of the classical COOK modulation which are resumed in the fact that it is one of the simplest modulation schemes. Indeed, its transceiver architecture has less complexity with a very low cost. In addition, it overcomes the main problem of classical COOK system resumed in the fact that this last requires nonzero constant decision threshold.
The transmitter of AT-COOK is composed of a binary information module, an UWB chaotic generator and a modulator (Multiplier). The receiver is composed of two Envelope Detector (ED) modules, for estimating separately the CRP energy and the noise energy, a subtractor and an Adaptive Threshold Estimator (ATE). At the transmitter and according to the COOK principle, the UWB chaotic carrier is modulated directly by binary information with bit duration $T_{b}$ and bit energy $E_{b}$. As it is shown in Fig.6, the bit "1" is represented by a CRP with duration $\tau_{c}=T_{b} / 2$ while the bit " 0 " is represented without CRP. At the receiver, after the propagation through an AWGN channel, each received noisy CRP of duration $T_{b}$ is devised into two parts of duration $T_{b} / 2$. They are treated separately by the CRP and Noise energy EDs, respectively. Thus, from the Eq.(6) and the fact that the chaotic and the noise signals are uncorrelated at the duration $T_{b} / 2$, we obtain at the output of the CRP-ED the estimated energy value $E_{b}+N_{0}$, where $N_{0}$ is the noise energy at the first part at duration $T_{b} / 2$, and at the output of the noise ED. Thereby, we obtain the estimated noise energy $N_{0}^{\prime}$ of the second part at duration $T_{b} / 2$.

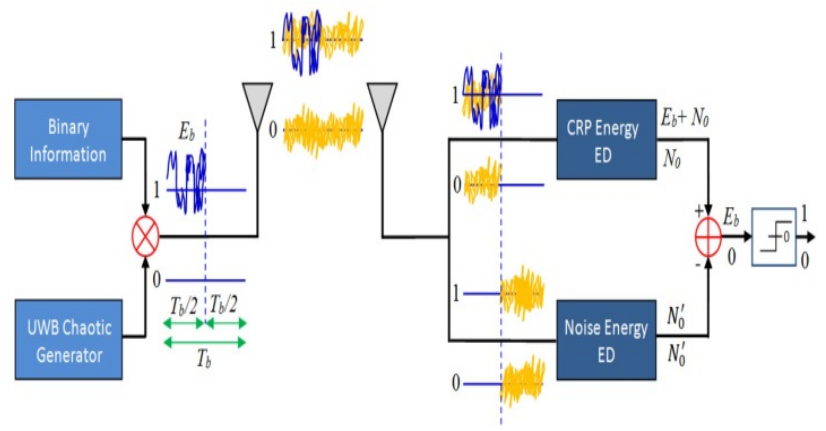

Fig. 6. AT-COOK principle for UWB DCC system.

Therefore, after the energies estimation and the subtraction operation, we obtain the following estimated energy values:

- $\quad$ For bit "1":

$\widehat{E}_{b}=E_{b}+N_{0}-N_{0}^{\prime}$

- For bit "0":

$\widehat{E}_{b}=N_{0}-N_{0}^{\prime}$

From the above equations, after the subtraction, we define the estimated threshold value as follows:

$\hat{d}=N_{0}-N_{0}^{\prime}$

which can be simplified by the following expression:

$\hat{d}=(\alpha-1) N_{0}^{\prime}$

with supposing that $N_{0}=\alpha N_{0}^{\prime}$, where $\alpha>0$ is a constant parameter taking a small values because $N_{0}^{\prime}$ and $N_{0}$ are not very different in the bit duration $T_{b}$.

The equation (10) shows that by affecting a constant value to the parameter $\alpha$, the estimated threshold depends only on the estimated noise energy $N_{0}^{\prime}$ of the second part, i.e., the decision threshold is adapted automatically to the estimated noise energy value. Consequently, in the general 
case, the decision with the adaptive threshold is defined as follows:

$$
\left\{\begin{array}{l}
\hat{d}>(\alpha-1) N_{0}^{\prime}, " 1 " \text { is transmitted } \\
\hat{d}=(\alpha-1) N_{0}^{\prime}, " 0 " \text { is transmitted }
\end{array}\right.
$$

However, since that in the UWB technology, the bit duration $T_{b}$ is of the order of ns, we can assume that at $90 \%$ of cases $N_{0} \approx \alpha N_{0}^{\prime}$ which means that $\alpha=0.9$. Thus, for example in the case of $N_{0}^{\prime}=N_{0}$, the threshold value of Eq. (11) will be equal to zero $(d=0$ with $\alpha=1)$ and then the decision with the zero threshold is given by:

$\left\{\begin{array}{l}\hat{d}>0, " 1 " \text { is transmitted } \\ \hat{d}=0, " 0 " \text { is transmitted }\end{array}\right.$

At this level, we can say that, the challenge is to choose the value of the parameter $\alpha$ which leads to better performance of the UWB Chaotic-OOK communication system.

\section{Simulation Results and Performance Analysis}

In this section, we present simulations and BER performance analysis, using the ADS simulator, for the UWB DCC system based on the proposed AT-COOK modulation. To show the good performance of the proposed non-coherent modulation technique, we compare its BER performance versus some well-known non-coherent techniques as COOK, CDSK, DCSK and RD-DCSK with choosing the decision threshold equal to 0 .

\subsection{Simulated AT-COOK UWB DCC system}

We simulate with ADS software the principle scheme of the UWB DCC system presented in Fig.6. The system is composed by three main blocks, the AT-COOK transmitter, the Channel block and the AT-COOK receiver. The details of these blocks are presented in the following subsections.

\subsubsection{AT-COOK Transmitter}

The ADS architecture of the AT-COOK transmitter is presented in Fig. 7. It is composed by three blocks, a Binary Information Generator (BIG), an UWB Chaotic Generator (UCG) and a COOK Modulator (CM). The UCG block is obviously detailed and simulated in Section 2 and the details of the two others are as follows:

- Binary Information Generator: The block contains three sub-blocks, a Data block which generates the information data signal at the Return-to-zero (RZ) data format with the bit duration $T_{b}$, a Clock block which fixes the Duty Cycle (DC) of the data and a Switcher which forms RZ data signal.

- COOK Modulator: The modulator of the COOK modulation technique is a multiplier. It realizes the direct multiplication of the binary information with the UWB chaotic carrier. Fig. 8 shows the direct modulation results of UWB chaotic carrier by information data with $D C=50 \%$ and $T_{b}=200 \mathrm{~ns}$.

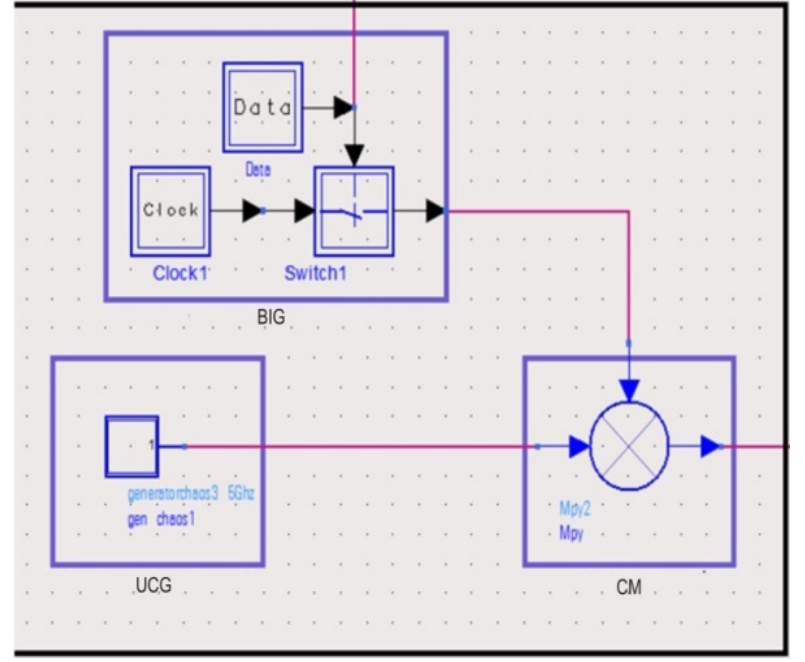

Fig. 7. DS architecture of the AT-COOK Transmitter.

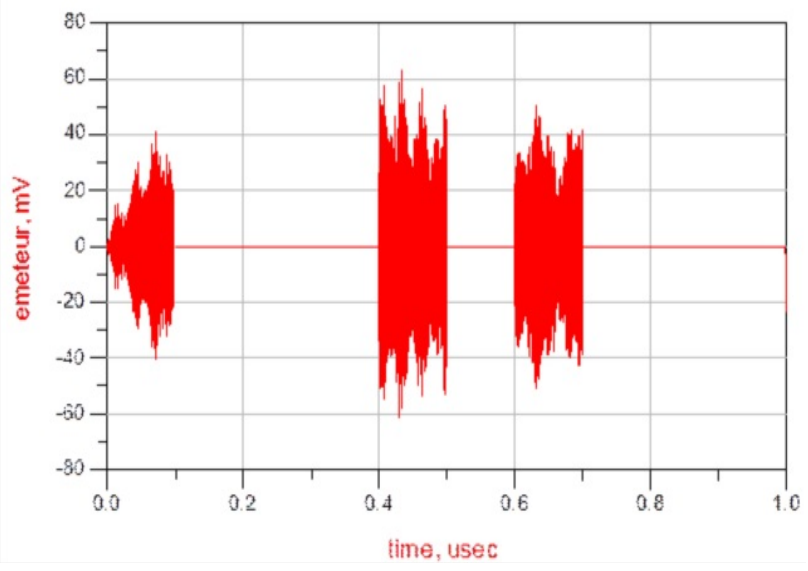

Fig. 8. CRP trains of UWB DCC COOK transmitter.

\subsubsection{Channel Block}

The ADS software proposes many types of transmission channel. We choose for our application the AWGN channel. Note that the goal of this work is to validate the proposed approach and further it is possible to study the performance of this technique using the ADS channel models such as Fading, UWB or WLAN channels. Fig.9 shows the model of the AWGN channel in ADS, where EbNO is the ratio value $E_{b} / N_{0}$.

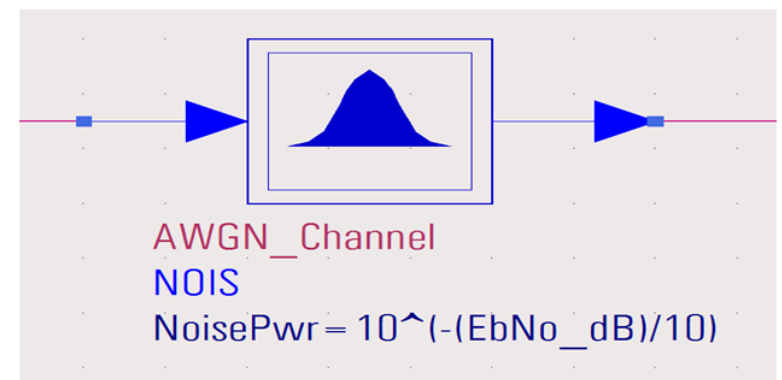

Fig. 9. ADS model of AWGN.

\subsubsection{AT-COOK Receiver}

The ADS architecture of the AT-COOK receiver, presented in the scheme of the UWB DCC system of Fig. 6, is shown 
in Fig. 10. The architecture is composed by three main blocks: an Information Correlator Block (ICB), a Noise Correlator Block (NCB) and a Decision Block (DB). In the following, we give the details of these blocks.

- Information Correlator Block: This block estimates the energy of the received signal at the first part of duration $T_{b} / 2$. For this, it separates the first part by multiplying the received signal by a clock signal of amplitude unity, $D C=50 \%$ and period $T_{b}$ fixed by the clock module. After, it multiplies the received signal by itself and then it integrates the result at the duration $T_{b} / 2$ by using a RF integrator (InDumpt) controlled by the corresponding clock module. Thus, the results of these two operations is the estimated energy at the duration $T_{b} / 2$.

- Noise Correlator Block: This block has the same architecture of that ICB, and uses the same operations to estimate the noise energy at the second part of each received bit at duration $T_{b} / 2$.

- Decision Block: This block takes the decision about the received bit by comparing the estimated energy to a predefined threshold. It contains two Switchers, controlled by clock modules, which control the decision time. A Subtractor which permits the estimation of the CRP energy by subtracting the estimated noise energy from the noised CRP energy and a Quantizer which compares the estimated CRP energy to an adaptive threshold. In the general case, this block depends on the estimated noise energy. However, in our case, i.e., UWB communication, we can fix the threshold zero as it is explained above.

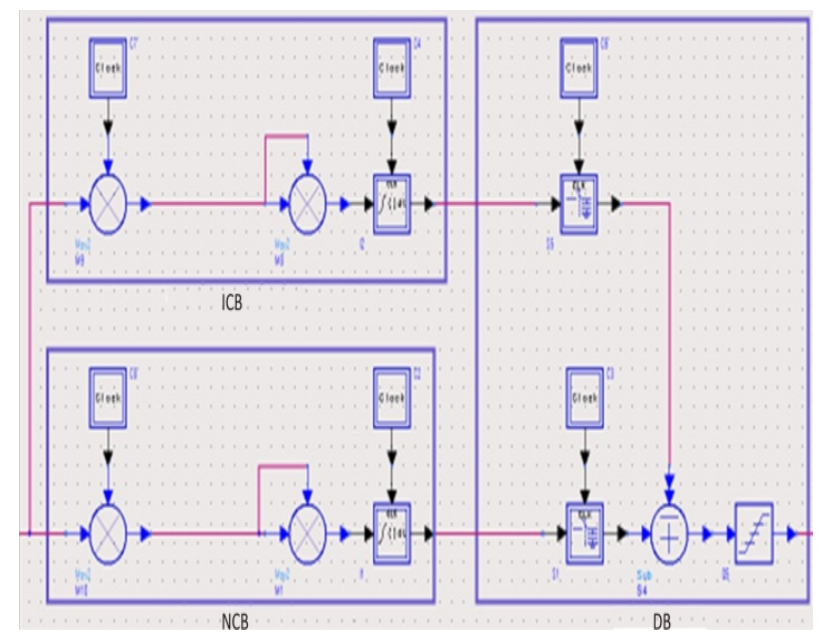

Fig. 10. ADS architecture of the AT-COOK receiver.

\subsection{Performance Analysis}

The BER performance result of the simulated UWB DCC system, based on the proposed AT-COOK modulation technique with zero decision thresholds, is presented in Fig.11. This result is obtained for the following parameter values: bit duration $T_{b}=200 \mathrm{~ns}, D C=50 \%$ and for 2500 information data bits. From this result, we note that with the proposed AT-COOK technique, the BER decrease with the ratio $E_{b} / N_{0}$ and for 2500 bits, the maximum BER $\approx 10^{-3}$ in the system is achieved at $E_{b} / N_{0}=6 \mathrm{~dB}$. This proves the good performances of the proposed AT-COOK approach combined with the UWB DCC technology.

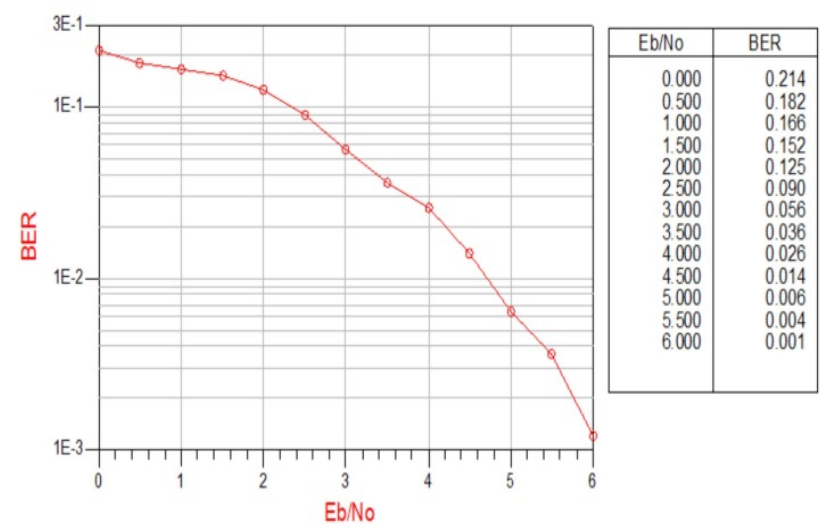

Fig. 11. BER performance of the AT-COOK applied in UWB DCC system.

To confirm and validate the above conclusion, we have compared the performance of our approach to those of the well-known non-coherent modulation techniques, which are largely investigated in the literature like classical COOK, CDSK, DCSK and RD-DCSK. For this purpose, we have simulated separately, under the same parameter values used above, the BER performance of each modulation technique applied lonely in the UWB DCC system. We superpose all of the curves of the performance results and present them in Fig.12. The obtained results show that the proposed ATCOOK presents good BER performance then the four simulated non-coherent modulation techniques when applied in an UWB DCC system. These good performances are the result of two factors. The first one is the AT-COOK receiver architecture, where the noise energy is estimated instantly and subtracted from the received CRP energy. The second one is resumed in the definition of an adaptive decision threshold and which can be chosen zero in the case of UWB DCC technology. Moreover, it should be noted that, the curves of the BER performances results presented in Fig.12 can be improved by using a large number of information bits, for example one million bits. However, in our case study, we are limited by the performance of the available HP workstation. In fact, the ADS software consumes huge memory space to store the calculated data. Nevertheless, since the results of Fig.12 clearly allow us to draw meaningful conclusions, we simply make the simulations with 2500 bits.

In addition, from the principle of the proposed ATCOOK technique, we can affirm that our approach:

1. Eliminates the threshold problem of the classical COOK which uses a constant nonzero decision threshold;

2. Provides a high throughput comparing with the others techniques since it do not uses any delay at the modulation;

3. Permits to economize the transmitted power, because the transmitted bit energy is devised by two (transmission only during $T_{b} / 2$ ). 


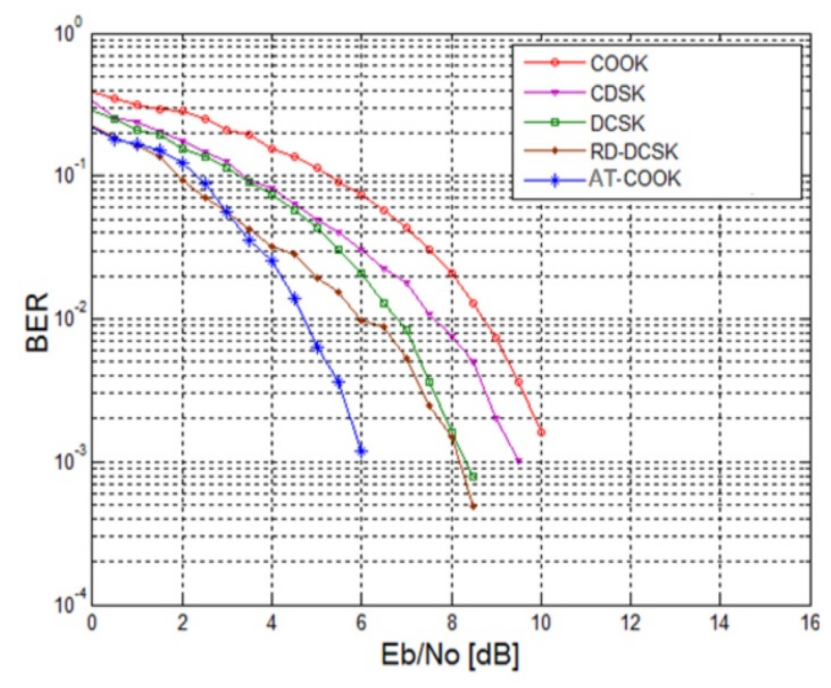

Fig. 12. BER performance of the proposed technique compared to existing methods.

Finally, thanks to the advantages and improvements provided by the proposed AT-COOK technique, we can conclude that among the non-coherent chaotic modulation techniques proposed until now, our technique is the best candidate for the UWB DCC technology.

\section{Conclusion}

In this paper, a new non-coherent chaotic modulation based on an adaptive decision threshold is proposed. Particularly, for the case of UWB Direct chaotic communication technology applications, it is possible to choose the decision threshold equal to zero because the estimated noise is almost equal to the real noise at the duration $T_{b} / 2$. With this zero decision threshold, our technique provides a good BER performance obtained by simulations with the ADS simulator. In addition, it presents the best BER performance than four well known noncoherent chaotic modulation techniques. Therefore, the proposed non-coherent chaotic modulation technique is an efficient solution for all DCC-based communication schemes. As perspective of this work, firstly, we plan to study the performance of the proposed technique according to the values of the parameter $\alpha$. Finally, realize experimental validation through RF communication of the proposed AT-COOK technique.

\section{References}

1. A. Panas, B. Kyarginsky, and N. Maximov, Single-transistor microwave chaotic oscillator, In. Proc. of NOLTA2000, Dresden, Germany, pp. 445-448 (2000).

2. N. Maximov, A. Panas, and S. Starkov, Chaotic oscillators design with preassigned spectral characteristics, In. Proc. of the ECCTD01-European Conference on Circuit Theory and Design, August 28-31, Espoo, Finland (2001).

3. G. Mykolaitis, A. Tamasevicius, and S. Bumeliene, Experimental demonstration of chaos from Colpitts oscillator in VHF and UHF ranges, Electronics Letters, vol. 40(2), pp. 91-92 (2004).

4. Z.G. Shi and L.X. Ran, Microwave chaotic Colpitts oscillator: design, implementation and applications, J. of Electromagn. Waves and Appl., vol. 20(10), pp. 1335-1349 (2006).

5. A. Tamasevicius, G. Mykolaitis, S. Bumeliene, A. Baziliauskas, R. Krivickas, and E. Lindberg, Chaotic Colpitts oscillator for the ultrahigh frequency range, Nonlinear Dynamics, vol. 44(1-4), pp. 159-165 (2006).

6. M.P. Kennedy, Chaos in the Colpitts oscillator, IEEE Trans. on Circuits and Sys., vol. 41(11), pp. 771-774 (1994).

7. A.S. Dmitriev, Kyarginsky, B. Ye., N.A. Maximov, A. I. Panas, and S. O. Starkov, Prospects of direct chaotic communications design in RF and microwave band, Radiotehnika, vol. 42(3), pp. 9-20 (2000).

8. Z.G. Shi, S. Qiao, and K.S. Chen, Ambiguity functions of the direct chaotic radar employing microwave chaotic Colpitts oscillator, Progress In Electromagnetics Research, vol. 77, pp. 114 (2007).

9. S. Qiao, Z.G. Shi, and K.S. Chen, A new architecture of UWB RADAR utilizing microwave chaotic signals and chaos synchronization, Progress In Electromagnetics Research, vol. 75, pp. 225-237 (2007).
10. A. Dmitriev, M. Hasler, A.I. Panas, and K.V. Zakharchenko, Basic principles of direct chaotic communications, Nonlinear Phenomena in Complex Systems, vol. 4(1) (2002).

11. A.S. Dmitriev, A.I. Panas, and S.O. Starkov, Direct chaotic communication in microwave band, Electronic Nonlinear Science Preprint, nlin.CD/0110047 (2001).

12. A. Dmitriev, B. Kyarginsky, A. Panas, and S. Starkov, Direct chaotic communication system experiments, In. Proc. of the NDES01 Conference Delft. Netherlands, pp. 157-160 (2001).

13. A.S. Dmitriev, B.Ye. Kyarginsky, A.I. Panas, and S.O. Starkov, Experiments on ultra wideband direct chaotic information transmission in microwave band, Int. J. Bifurcation Chaos, vol. 13(6), 1495-1507 (2003).

14. C.C. Chong, and S.K. Yong, UWB direct chaotic communication technology for low-rate WPAN applications, IEEE Transactions on Vehicular Technology, vol. 57(3) (2008).

15. Advanced Design System (ADS), Agilent EEsof EDA home page.

16. G. Kolumban, B. Vizvari, W. Schwarz, and A. Abel, Differential chaos shift keying: A robust coding for chaos communications, In. Proc. of the 4th Int. Workshop on Nonlinear Dynamics of Electronics Systems, (NDES96), Seville, Spain, pp.87-92 (1996).

17. M. Sushchik, S. Tsimring, and R. Volkovskii, Performance analysis of correlation-based communication schemes utilizing chaos, IEEE Trans. Circ. Syst.-I., vol. 47(12) (2000).

18. K. Lee, J. Son, J. Kim, Y. Kim, and H. Park, Performance analysis of the reduced-delay DCSK UWB System, In. Proc. of the 21st International Technical Conference on Circ. Syst. Comput. Comm., ISBN 974-944189-3, ECTI, pp. 137-140 (2006). 